\title{
Global Voices: Crossing Borders in Automatic News Summarization
}

\author{
Khanh Nguyen ${ }^{\odot}$ and Hal Daumé III ${ }^{\odot} \odot$

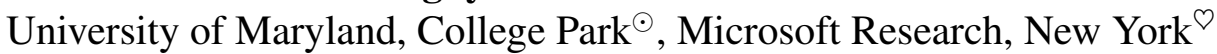 \\ $\{$ kxnguyen, hal $\}$ eumiacs.umd.edu
}

\begin{abstract}
We construct Global Voices, a multilingual dataset for evaluating cross-lingual summarization methods. We extract social-network descriptions of Global Voices news articles to cheaply collect evaluation data for intoEnglish and from-English summarization in 15 languages. Especially, for the into-English summarization task, we crowd-source a highquality evaluation dataset based on guidelines that emphasize accuracy, coverage, and understandability. To ensure the quality of this dataset, we collect human ratings to filter out bad summaries, and conduct a survey on humans, which shows that the remaining summaries are preferred over the social-network summaries. We study the effect of translation quality in cross-lingual summarization, comparing a translate-then-summarize approach with several baselines. Our results highlight the limitations of the ROUGE metric that are overlooked in monolingual summarization.
\end{abstract}

\section{Introduction}

Cross-lingual summarization is an important but highly unexplored task. The ability to summarize information written or spoken in any language at a large scale would empower humans with much more knowledge about the diverse world. Despite the fast development of automatic summarization (Allahyari et al., 2017; Dong, 2018; Gambhir and Gupta, 2017), present technology mostly focuses on monolingual summarization. There is currently lacking a standard, high-quality multilingual dataset for evaluating cross-lingual summarization methods. Two main challenges present in constructing such a dataset. First, the cost of crowsourcing human-written summaries is high. It generally takes a long time for a human to summarize a document, as they not only have to read and understand information in the article, but also have to

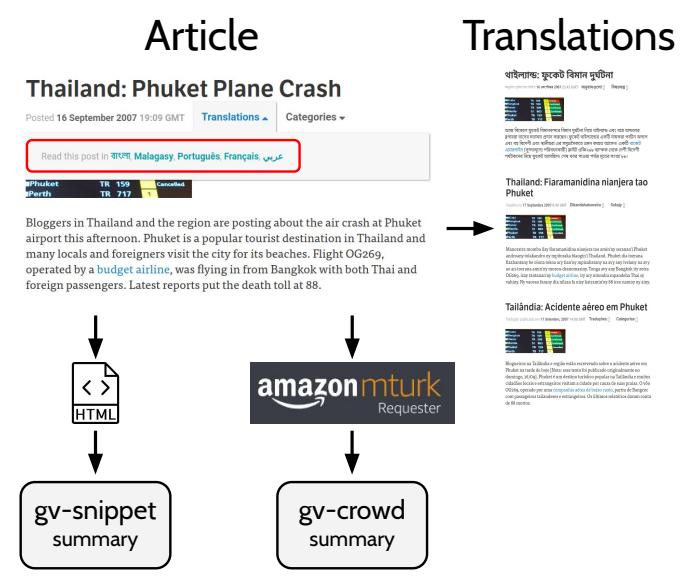

Figure 1: Data construction pipeline. We collect two types of summary: (a) the social network description of the article (gv-snippet) and (b) the 50-word summary written by Mechanical Turk workers following our guidelines (gv-crowd).

make complex decisions in sieving and paraphrasing the information. Second, it is difficult to design summarization guidelines for humans, as the task is generally not well-defined: the selection of what content is "important" in a summary is based on subjective and common-sense rules that vary among individuals and are difficult to be expressed precisely in words.

Even in monolingual summarization, there were limited attempts in constructing summarization datasets via crowd-sourcing (Over et al., 2007; Dang and Owczarzak, 2008, 2010). These datasets are mostly used for evaluation due to their small sizes. To construct large-scale training datasets, researchers mine news sources that naturally provide human-written summaries (Hermann et al., 2015; Sandhaus, 2008), or construct artificial summaries from document titles (Rush et al., 2015). Summaries collected in this way may be not best for evaluation because they are generated under unknown guidelines (or there may be no guide- 
lines at all). Previous work on cross-lingual summarization performs evaluation with human judgments (Orsan and Chiorean, 2008), or with automatic metrics and noisy source articles generated by automatic translation systems (Wan et al., 2010; Ouyang et al., 2019). The former approach is expensive and not reproducible, while the latter is prone to biases induced by translation systems that could be further amplified by summarization systems.

This paper presents Global Voices, a highquality multilingual dataset of summaries of news articles. The dataset can serve as a standard benchmark in both multilingual and cross-lingual summarization. Global Voices ${ }^{1}$ is a multilingual website that reports and translates news about unheard voices across the globe. Translation in this website is performed by the Lingua team, ${ }^{2}$ consisting of volunteer translators. As of August 2019, Global Voices provides translations of news articles in 51 languages; many articles are translated into multiple languages. Figure 1 illustrates a sample article from Global Voices. We extract the socialnetwork descriptions of the articles to (cheaply) construct gv-snippet, an evaluation set for multilingual and cross-lingual news summarization. Nevertheless, these descriptions usually have poor coverage over the original contents because they were written with the intention of drawing user clicks to read more about the articles. Therefore, besides gv-snippet, we construct a smaller but higher-quality dataset of human-written English summaries, called gv-crowd, based on our guidelines which explicitly emphasize accuracy, coverage and understandability. The Global Voices dataset is summarized in Table 2. It currently supports 15 languages, which span nine language genera (Romance, Barito, Indic, Slavic, Semitic, Greek, Germanic, Japanese, Bantoid) and five language families (Indo-European, Austronesian, Japanese, Niger-Congo, Afro-Asiatic).

\section{Dataset Construction}

Data Collection and Pre-Processing. Using Scrapy, ${ }^{3}$ we crawl and download HTML source codes of 41,939 English articles and their translations. We use bs $4^{4}$ to extract each article's main

\footnotetext{
1https://globalvoices.org/

2 https://globalvoices.org/lingua/

3 https://scrapy.org/

4 https://www.crummy.com/software/Beautifulsoup/
}

\begin{tabular}{|c|c|c|c|}
\hline Language & ISO 639-1 & gv-snippet & gv-crowd \\
\hline \multicolumn{4}{|c|}{ Number of articles } \\
\hline English & en & 4,573 & 529 \\
\hline Spanish & es & 3,921 & 487 \\
\hline Malagasy & $\mathrm{mg}$ & 2,680 & 374 \\
\hline Bengali & bn & 2,253 & 352 \\
\hline French & $\mathrm{fr}$ & 2,130 & 352 \\
\hline Portuguese & $\mathrm{pt}$ & 798 & 162 \\
\hline Russian & $\mathrm{ru}$ & 795 & 139 \\
\hline Arabic & ar & 745 & 191 \\
\hline Italian & it & 718 & 135 \\
\hline Macedonian & $\mathrm{mk}$ & 701 & 138 \\
\hline Greek & el & 694 & 128 \\
\hline German & de & 647 & 204 \\
\hline Japanese & ja & 424 & 75 \\
\hline Swahili & sw & 418 & 84 \\
\hline Dutch & $\mathrm{nl}$ & 348 & 87 \\
\hline \multicolumn{4}{|c|}{ Other statistics } \\
\hline \multicolumn{2}{|c|}{ Summarized by } & GV authors & MTurkers \\
\hline \multicolumn{2}{|c|}{ Summary languages } & All versions & English \\
\hline \multicolumn{2}{|c|}{ Summary lengths (words) } & - & $40-50$ \\
\hline \multicolumn{2}{|c|}{ Article lengths (words) } & $150-500$ & $150-350$ \\
\hline
\end{tabular}

Table 1: Summary of the Global Voices dataset. The dataset include articles in 15 languages. English versions of all non-English articles are included. The gv-snippet split contains social-network summaries of all articles, while the gv-crowd split contains crowd-sourced summaries of English articles.

content and remove image captions. Next, we use html2text ${ }^{5}$ to convert the main content's HTML source code to regular text, removing web-page and image URLs. Since an article may content block-quotes written in original languages, we detect language of each paragraph and remove paragraphs that are not in the article's main language. Language detection is conducted by voting decisions of four packages: langdetect, ${ }^{6}$ langid, ${ }^{7}$ polyglot, ${ }^{8}$ fastText $^{9}$ (Joulin et al., 2016a,b).

Constructing gv-snippet. This split includes articles whose English versions contain from 150 to 500 words. For each article, we extract its Open Graph description by extracting the meta tag with property og:description in the HTML source code, and use the description as the reference summary of the article. These descriptions are short text snippets that serve as captions of the articles when they appear on social networks (e.g. Facebook, Twitter).

Crowd-sourcing gv-crowd. We select English

\footnotetext{
5 https://pypi.org/project/html2text/

${ }^{6}$ https://pypi.org/project/langdetect/

${ }^{7}$ https://github.com/saffsd/langid.py

8 https://pypi.org/project/polyglot/

${ }^{9}$ https://fasttext.cc/docs/en/

language-identification.html
} 
articles that contain 150-350 words, and request workers from Mechanical Turk ${ }^{10}$ (MT) to summarize them in 40-50 words. Each HIT $^{11}$ asks a worker to summarize five articles in 35 minutes. We recruit Turkers in Canada and the U.S.A. with Masters qualification, a HIT approval rate greater than or equal to $97 \%$, and a number of HITs approved greater than or equal to 1,000 . On average, collecting a summary costs 1.50 USD (including taxes and extra fees). We inform workers of our evaluation guidelines, which focus on three criteria:

- Accuracy: information in a summary should be based on the original article only. It can be paraphrased from but should not disagree with information in the article.

- Coverage: a summary should reflect the most important messages/stories in the original article. Each message/story should be captured as detailed as possible, without missing other important messages/stories.

- Understandability: a summary must be written in standard, fluent English. Readers must be able to understand the summary without reading the original article. Understanding the summary must not require any additional knowledge beyond knowledge required to understand the article.

In comparison, the DUC-2004 dataset (Over et al., 2007) only provides subtle format suggestions and leaves the summary contents almost entirely to the decisions of the writers:

\begin{abstract}
"...Imagine that to save time, rather than read through a set of complete documents, you first read a list of very short summaries of those documents and based on these summaries you choose which documents to read in their entirety. Create your very short summaries to be useful in such a scenario. A very short summary could look like a newspaper headline, be a list of important terms or phrases separated by commas, a sentence, etc. It should not contain any formatting, i.e., no indented lists, etc. Feel free to use your own words."
\end{abstract}

Source: https://duc.nist.gov/duc2004

Our guideline criteria are similar to those of the TAC 2010's guided summarization task (Dang and Owczarzak, 2010) but we do not restrict the summary format using domain-specific templates.

\footnotetext{
${ }^{10}$ https://www. mturk.com/

${ }^{11}$ a Mechanical Turk task.
}

Some articles may read disrupted due to removals of images and videos, and may contain non-English texts. To ensure the summaries are based on the English texts only, we advise workers to (a) not web-search for the original content and (b) ignore the non-English contents. We also emphasize spelling words correctly and recommend copying difficult-to-spell words from the original articles. In the end, we collect 840 summaries for 738 articles.

Human Evaluation of gv-crowd. The summarycollecting task receives mostly positive feedback from workers. The task is widely regarded as "fun", "interesting", and "challenging". However, many workers raised concern about the strict time constraint. To evaluate the quality of the dataset, we launch another MT task in which we ask workers to rate and post-edit the summaries collected in the previous task. Each task HIT requires evaluating ten summaries in 60 minutes. We recruit workers in Canada and the U.S.A. with a HIT approval rate greater than or equal to $97 \%$, and a number of HITs approved greater than or equal to 1,000 .

Specifically, we ask workers to provide two types of ratings: criterion-based ratings and overall ratings. Each worker is instructed to first give a 1-to-5 rating of a summary in each of our three criteria (accuracy, coverage, understandability), and then to give an overall rating of the summary. We define three levels of the overall rating:

- Bad: the summary misrepresents the original article. It contains factual errors that disagree with the content of the article. OR it does not cover the most important message/story of the article. OR it is missing other important points that could easily be included without violating the 50 -word constraint.

- Acceptable: the summary covers the most important message/story of the article. It does not contain factual errors. It is missing one or two important points that would be difficult to include in a 50-word summary.

- Good: the summary covers the most important message/story of the article. It does not contain factual errors. All important points are captured.

In addition, the worker is required to write short reasons (each in 5-25 words) to justify their ratings. 
Among 840 summaries collected, 383 (45.60\%) were rated as Good, 264 (31.43\%) Acceptable, and $193(22.98 \%)$ Bad. We observe that among the three criteria, understandability is easiest to meet while coverage is the most challenging: the mean understandability rating is 4.06 while the mean coverage rating is only 3.47 ; about $90 \%$ of the summaries attain understandability ratings of at least 3. By computing Pearson correlation coefficients, we find that the overall rating most strongly linearly correlates with the coverage rating $(0.81)$ and least with the understandability rating (0.57). Common flaws identified by the human evaluators include: missing important points, factual errors, abstruse and/or verbose writing.

To construct the gv-crowd split, we pair each article with its highest-rated summaries ${ }^{12}$ and excluded articles that (a) are paired with Bad summaries or (b) have a criterion-based rating below 3 . We also ask workers to correct spelling and factual errors in the Bad summaries, but these postedited summaries require further evaluation to be included in the dataset in the future. To facilitate summarization evaluation studies, we will release all the summaries accompanied with their ratings, reasons, and post-edit versions.

For a (randomly selected) subset of 50 articles, we collect three summaries per article to study the diversity in quality and language usage among human-written summaries of the same documents. We find that the summary quality does not vary greatly: the overall-rating difference between the highest and lowest rated summaries is at most 1 in $74 \%$ of these articles. To quantify the diversity of summaries, we calculate the pairwise RoUGE scores, using one summary as the reference and another as the predicted

RoUGE $_{\text {pair }}=\frac{1}{3 \cdot 50} \sum_{i=1}^{50} \sum_{1 \leq j<k \leq 3} \operatorname{RougE}\left(s_{i, j}, s_{i, k}\right)$

where $s_{i, j}$ and $s_{i, k}$ are distinct summaries of the $i$-th article. The RougE pair $^{-1,2, L ~ F-1 ~ s c o r e s ~ a r e ~}$ relatively low $(39.44,12.39$, and 32.85 , respectively), indicating that the summaries highly vary in vocabulary and sentence structure.

\footnotetext{
${ }^{12}$ For a pair of summaries, we first compare their overall ratings, then sums of three criterion-based ratings, then the individual accuracy, coverage, understandability ratings (in this specific order).
}

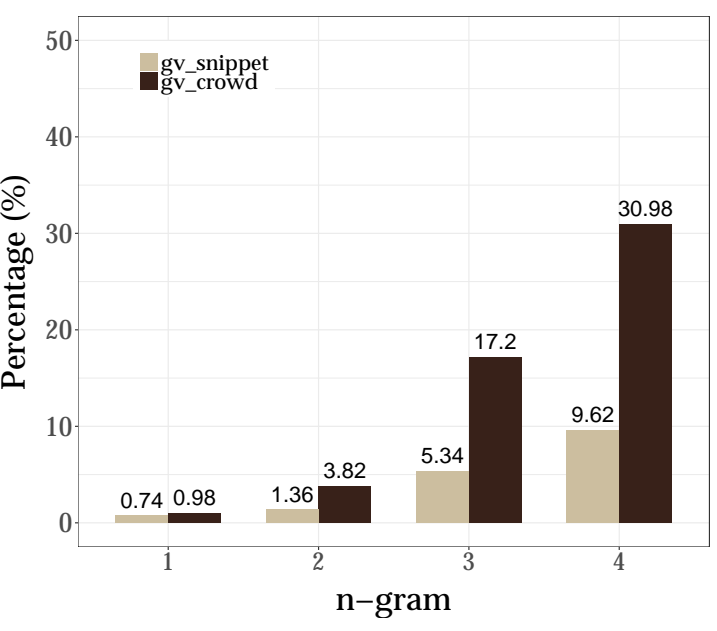

Figure 2: Average fraction of $n$-grams in the summary that are not seen in the original article.

Human Comparison of gv-snippet and gv-crowd. To ensure that the gv-crowd summaries are of higher quality than the gv-snippet summaries, we conduct a survey that asks MT workers to compare the two types of summary. Concretely, each worker reads an article and its gv-snippet and gv-crowd summaries. We ask the worker to specify which summary (or none) is better in each of the three criteria and is better overall. We remove partial sentences that end with "..." in the gv-snippet summaries to ensure that the workers rate the two types of summary mainly based on their contents, not based on any peculiar features. We also randomly shuffle the order of the summaries in a pair so that the workers cannot rely on the order to determine the summary type. Each worker is given 45 minutes to compare five summary pairs. Each summary pair is evaluated by three workers. We recruit workers with similar qualifications to those in the gv-crowd evaluation task.

The outcome of this survey is positive. In 22 out of the 30 articles included in the survey $(75.9 \%)$, at least 2 out of 3 workers prefer the gv-crowd summary. Overall, 63 out of 90 workers (70.0\%) prefer the gv-crowd summaries to its gv-snippet counterparts. As expected, coverage is the criterion where the gv-crowd summaries show most strength against the gv-crowd summaries, with a preference ratio of $83.3 \%$ (25/30) compared to $66.7 \%(20 / 30)$ of accuracy or understandability.

We also evaluate these two types of summary in terms of how novel their summaries are compared to the original articles. Figure 2 shows the 


\begin{tabular}{|c|c|c|}
\hline Model & Train & Validation \\
\hline \multicolumn{3}{|c|}{ Translation (sentences) } \\
\hline Spanish-English & $4.1 \mathrm{M}$ & $3 \mathrm{~K}$ \\
\hline French-English & $5.6 \mathrm{M}$ & $3 \mathrm{~K}$ \\
\hline German-English & $151.6 \mathrm{~K}$ & $2 \mathrm{~K}$ \\
\hline Arabic-English & $174.3 \mathrm{~K}$ & $2 \mathrm{~K}$ \\
\hline \multicolumn{3}{|c|}{ Summarization (pairs of documents and summaries) } \\
\hline English & $287.2 \mathrm{~K}$ & $13.4 \mathrm{~K}$ \\
\hline
\end{tabular}

Table 2: Data used to train and validate translation and summarization models.

average fractions of novel $n$-grams of each type of summary. Overall, the summaries reuse most words in the articles. The gv-crowd summaries contain substantially more novel 3 -grams and 4grams than the gv-snippet summaries, partly because each sentence of a gv-crowd summary usually includes information from multiple sentences in the original article. On $73 \%$ of the articles in the gv-crowd split, the gv-crowd summary has higher fractions of novel $n$-grams than the gv-snippet counterpart (with $n=1,2,3,4$ ).

\section{Experiments}

We study the task of generating English summaries of non-English news articles. This task can naturally be decomposed into two subtasks: translation and summarization. We follow a translatethen-summarize approach where each article is first translated into English using a pre-trained machine translation model, then the translation is summarized using a pre-trained English summarization model. Data for training models in both subtasks are publicly available, allowing solving the joint task in a zero-shot manner, in the sense that no parallel pairs of (original document, English summary) are provided during training. On the other hand, a summarize-then-translate approach is practically difficult to implement because of the lack of large-scale datasets for training reliable summarization models in non-English languages.

Translation models. Our goal is to study the effect of translation quality in this task. Hence, we employ translation models trained under various amounts of resources. We conduct experiments in four source languages: Spanish (es), French (fr), German (de), and Arabic (ar). Concretely, we train the $\{$ es,fr $\}$-en models using the large-scale CommonCrawl and News Commentary datasets, and train the $\{\mathrm{de}$, ar $\}$-en models using the low-resource multilingual TED (Duh, 2018) dataset. We apply standard machine translation pre-processing steps, normalizing and tokenizing the data with Moses scripts. We tokenize Arabic texts with the PyArabic tool (Zerrouki, 2010). Our translation models implement the Transformer architecture (Vaswani et al., 2017). The $\{$ es,fr $\}$-en models have the same hyperparameters as those of the base Transformer architecture described in Table 3 of Vaswani et al. (2017). The $\{d e, a r\}$-en models have less parameters, using 4 attention heads and a feed-forward hidden size of 1024 . We train the models using the fairseq-py toolkit (Ott et al., 2019). Since the models are trained to perform sentence-level translation, we split the source articles into sentences, perform translation, and join the output sentences into articles. The training settings are the same as those of Vaswani et al. (2017) except that: (a) the maximum tokens in a batch is 4,000 , (b) the $\{$ es,fr $\}$-en models and the $\{$ de,ar $\}$-en models are trained for $5 \cdot 10^{4}$ and $8 \cdot 10^{5}$ iterations, respectively, and (c) the $\{$ de,ar $\}$-en models use a dropout ratio of 0.3 . Training with an Nvidia Ti$\tan$ Xp GPU took place in approximately 5 hours for the smaller models and 3.5 days for the larger models.

Summarization models. We employ the state-ofthe-art Bi-LSTM bottom-up abstractive summarization model (Gehrmann et al., 2018). We make use of a pre-trained instance of this model provided by OpenNMT-py (Klein et al., 2017) and trained on the CNN/DailyMail dataset (Hermann et al., 2015).

Baselines. We compare the following approaches:

- First50: copies the first 50 words of the English version of the source article.

- PerfectTrans: directly summarizes the English version of the source article.

- TRAnsThenSum: our approach which first translates the source article into English then summarizes the translation.

Evaluation. Translation quality is measured by corpus-level BLEU, treating each article as a data point. Summarization quality is determined by computing Rouge-1, Rouge-2, Rouge-L F-1 scores.

Results. Table 3 presents our results. A qualitative example is illustrated in Figure 3. As expected, translation quality varies among different pairs of languages. The Spanish-English model 


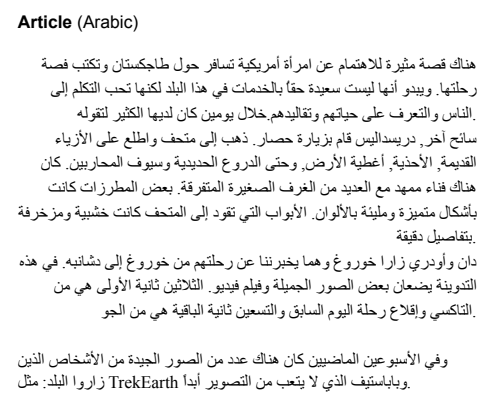

System summary (English)

The first treasure is from tennessee, and the last 90 seconds is from the atmosphere. In the last two weeks, there were a number of good photographs of people who babysitting that doesn't play from photography.
System Translation (English)

And it looks like it's not really happy to have services in this country, but she loves to talk to people and learn about their lives and their traditions. In two days she had a lot to say. Another driver, dallas, has visited a siege. Cyrus went to a museum and looked at the old physics of the old, the shoes, the warriors would stand. There was a temporary building with a lot of very small crafts. Some of the hamps were distinct and colorful. The doors that drive online were woode and preoccupied with a minute detail.

Dan and greenland, and they tell us about their journey from the elderly -lsb- unclear -rsb- rahmadan -lsb- unclear -rsb- . In this monitor, they put some beautiful images into a video. Thirty seconds ago, the first treasure is from tennessee, and the last journey of the day and the last 90 seconds is from the atmosphere.

And in the last two weeks, there were a number of good raphs of people who visited the country: like the treasures of climate and babysitting that doesn't play from photography.

gv_snippet summary (English)

There is interesting story of an American woman who travels around Tajkistan and writes a travelogue. It seems like she is not really happy with the service in this country but she traditions. In two days she had so.

\section{Reference Translation (English)}

There is interesting story of an American woman who travels around Tajikistan and writes a travelogue. It seems like she is not really happy with the service in this country but she loves to talk so much to say ...

Another tourist, @drysdales visited Hissar. He went to a museum and looked at old costumes, footwear, earthen wear and even the chain mail and sword of a warrior. There was a paved courtyard was in interesting and colourful sesigns. The doors leading into the museum were wooden and carved with detail.

Dan and Audrey visited Khorog and they are telling us about their flight from Khorog to Dushanbe. In they post they have some good photos and a video with the first 30 seconds from the taxi and takeoff of the previous day's flight and next 90 seconds are mid-air.

Also the past two weeks there were made some good shots by people who visited the country: TrekEarth (Saghirdasht pass) and babasteve who never gets tired of photographing.

gv_crowd summary (English)

This is about American woman who travels around Tajikistan and writes a travelogue. A certain woman does not appear to be happy wih the service in said country. Another person wrote about the Hissar museum and how old things looked.

Figure 3: An example in our dataset. The source document is originally written in English and is translated into Arabic by a Global Voices translator. Our translation system translates the Arabic article into English poorly. The summarization system mostly copies segments from the translation and carries grammatical errors (underlined) from the translation to its summary. The gv-snippet summary is a mere copy of the first few sentences of the English version of the article (though this may not always be the case in other articles). On the other hand, the gv-crowd summary offers better coverage, including information in the second paragraph. Note that this article is challenging to summarize perfectly in 50 words because it features four different parallel stories at the same time. Here, the gv-crowd summarizer trades off coverage for specificity of the stories.

\begin{tabular}{|c|c|c|c|c|c|c|c|c|c|c|c|c|}
\hline Method & \multicolumn{3}{|c|}{ Spanish-English } & \multicolumn{3}{|c|}{ French-English } & \multicolumn{3}{|c|}{ German-English } & \multicolumn{3}{|c|}{ Arabic-English } \\
\hline \multicolumn{13}{|c|}{ Translation quality (BLEU $\uparrow)$} \\
\hline Transformer & & 37.45 & & & 29.80 & & & 19.34 & & & 10.77 & \\
\hline \multicolumn{13}{|c|}{ Summarization quality evaluated on gv-snippet (RoUGE- $1|2| \mathrm{L}$ F-1 scores $\uparrow$ ) } \\
\hline FIRST50 & 63.7 & | $55.1 \mid$ & 61.3 & 64.7 & $|56.2|$ & 62.3 & 65.2 & | $57.1 \mid$ & & 62.9 & 53.5 & 60.5 \\
\hline PERF & 38.0 & 22.1 & 34.0 & 38.1 & 21.8 & 34.0 & 37.7 & 21.9 & 33.6 & 36.8 & 20.0 & 32.7 \\
\hline TRANSTHENSUM & 33.0 & 12.4 & 28.4 & 32.0 & 10.6 & 27.2 & 28.3 & 7.4 & 23.7 & 24.5 & 4.3 & 20.4 \\
\hline \multicolumn{13}{|c|}{ Summarization quality evaluated on gv-crowd (ROUGE-1|2|L F-1 scores $\uparrow$ ) } \\
\hline FIRST50 & 46.4 & | 23.4 | & 40.4 & 46.0 & $\mid 22.8$ | & 40.1 & 47.4 & $|25.7|$ & 40.9 & 45.9 & 22.9 & 40.4 \\
\hline PERFECTTRAI & 36.1 & 13.5 & 31.3 & 36.7 & 13.7 & 31.7 & 36.6 & 14.1 & 31.6 & 36.9 & 14.0 & 31.9 \\
\hline TransThenSum & 35.1 & $\mid 10.6$ & 30.0 & 33.3 & 8.9 & 28.5 & 29.4 & 6.0 & 25.0 & 26.0 & 3.8 & 22.1 \\
\hline
\end{tabular}

Table 3: Cross-lingual summarization results with different approaches. Translation quality is measured on the gv-snippet articles, of which the gv-crowd articles are a subset.

achieves the highest BLEU score (34.45) due to the amount of training data and the closeness between the language pair; on the other spectrum, the Arabic-English model offers poorest translations (10.77). Nevertheless, despite the large gaps in BLEU scores, we observe much smaller divergences in Rouge- 1 and Rouge-L scores. For example, in the extreme case of Arabic-English, even when the BLEU drops by almost $90 \%$ when switch from the reference to the predicted translations, the RoUGE-L F1-score only decreases by only about $30 \%$. This observation highlights a ma- jor limitation of RoUGE-1 and ROUGE-L: their insensitivity to the summary readability. Even though a source document may contain meaningless, ungrammatical contents (reflected by a low BLEU score), a model that summarizes by simply copying phrases can easily achieve high RoUGE1 and RougE-L scores. This limitation is difficult to observe in the context of monolingual summarization because the source documents come from natural sources and thus are mostly grammatical and meaningful. Another interesting finding is that the FIRST50 baseline achieves higher ROUGE 
scores when evaluated on gv-snippet than on gv-crowd. This observation indicates that the gv-snippet summaries overlap highly with the beginning part of the articles, confirming the results from our human preference survey that these summaries generally have poorer coverage over the entire articles than the $\mathrm{gv}$-crowd summaries.

\section{Conclusion}

This work introduces a dataset for evaluating multilingual and cross-lingual summarization methods in multiple languages. Future work aims to extend the dataset to more languages and construct a large-scale training dataset. Another interesting direction is to study whether multi-task learning can benefit cross-lingual summarization. To take advantage of the fact that translating the entire source article may not be necessary, it would be useful to teach models to devise more efficient translation strategies by informing them of the downstream summarization objective.

\section{Acknowledgement}

This material is based upon work supported by the National Science Foundation under Grant No. IIS1618193. Any opinions, findings, and conclusions or recommendations expressed in this material are those of the author(s) and do not necessarily reflect the views of the National Science Foundation.

\section{References}

Mehdi Allahyari, Seyedamin Pouriyeh, Mehdi Assefi, Saeid Safaei, Elizabeth D Trippe, Juan B Gutierrez, and Krys Kochut. 2017. Text summarization techniques: a brief survey. International Journal of Advanced Computer Science and Applications(IJACSA).

Hoa Trang Dang and Karolina Owczarzak. 2008. Overview of the tac 2008 update summarization task. In $T A C$.

Hoa Trang Dang and Karolina Owczarzak. 2010. Overview of tac 2010 summarization track. In TAC.

Yue Dong. 2018. A survey on neural networkbased summarization methods. arXiv preprint arXiv:1804.04589.

Kevin Duh. 2018. The multitarget ted talks task. http://www.cs.jhu.edu/ kevinduh/a/ multitarget-tedtalks/.

Mahak Gambhir and Vishal Gupta. 2017. Recent automatic text summarization techniques: a survey. $A r$ tificial Intelligence Review, 47(1):1-66.
Sebastian Gehrmann, Yuntian Deng, and Alexander M Rush. 2018. Bottom-up abstractive summarization. In Proceedings of Emperical Methods in Natural Language Processing.

Karl Moritz Hermann, Tomas Kocisky, Edward Grefenstette, Lasse Espeholt, Will Kay, Mustafa Suleyman, and Phil Blunsom. 2015. Teaching machines to read and comprehend. In Advances in neural information processing systems, pages 16931701.

Armand Joulin, Edouard Grave, Piotr Bojanowski, Matthijs Douze, Hérve Jégou, and Tomas Mikolov. 2016a. Fasttext.zip: Compressing text classification models. arXiv preprint arXiv:1612.03651.

Armand Joulin, Edouard Grave, Piotr Bojanowski, and Tomas Mikolov. 2016b. Bag of tricks for efficient text classification. arXiv preprint arXiv:1607.01759.

Guillaume Klein, Yoon Kim, Yuntian Deng, Jean Senellart, and Alexander M Rush. 2017. Opennmt: Open-source toolkit for neural machine translation. In Proceedings of the Association for Computational Linguistics.

C Orsan and Oana Andreea Chiorean. 2008. Evaluation of a cross-lingual romanian-english multidocument summariser. In International Language Resources and Evaluation.

Myle Ott, Sergey Edunov, Alexei Baevski, Angela Fan, Sam Gross, Nathan Ng, David Grangier, and Michael Auli. 2019. fairseq: A fast, extensible toolkit for sequence modeling. In Conference of the North American Chapter of the Association for Computational Linguistics.

Jessica Ouyang, Boya Song, and Kathleen McKeown. 2019. A robust abstractive system for cross-lingual summarization. In Proceedings of the 2019 Conference of the North American Chapter of the Association for Computational Linguistics: Human Language Technologies, Volume 1 (Long and Short Papers), pages 2025-2031.

Paul Over, Hoa Dang, and Donna Harman. 2007. Duc in context. Information Processing \& Management, 43(6):1506-1520.

Alexander M Rush, Sumit Chopra, and Jason Weston. 2015. A neural attention model for abstractive sentence summarization. In Proceedings of Emperical Methods in Natural Language Processing.

Evan Sandhaus. 2008. The new york times annotated corpus. Linguistic Data Consortium.

Ashish Vaswani, Noam Shazeer, Niki Parmar, Jakob Uszkoreit, Llion Jones, Aidan N Gomez, Łukasz Kaiser, and Illia Polosukhin. 2017. Attention is all you need. In Advances in neural information processing systems, pages 5998-6008. 
Xiaojun Wan, Huiying Li, and Jianguo Xiao. 2010. Cross-language document summarization based on machine translation quality prediction. In Proceedings of the 48th Annual Meeting of the Association for Computational Linguistics, pages 917-926. Association for Computational Linguistics.

Taha Zerrouki. 2010. pyarabic, an arabic language library for python. 